\title{
Ectopic Expression in the Giant Fiber System of Drosophila Reveals Distinct Roles for Roundabout (Robo), Robo2, and Robo3 in Dendritic Guidance and Synaptic Connectivity
}

\author{
Tanja A. Godenschwege, ${ }^{1}$ Julie H. Simpson, ${ }^{2}$ Xiaoliang Shan, ${ }^{1}$ Greg J. Bashaw, ${ }^{2}$ Corey S. Goodman, ${ }^{2}$ and \\ Rodney K. Murphey ${ }^{1}$ \\ ${ }_{1}^{1}$ Department of Biology, Morrill Science Center, University of Massachusetts, Amherst, Massachusetts 01003, and \\ 2Howard Hughes Medical Institute, Department of Molecular and Cell Biology, University of California, Berkeley, \\ California 94720
}

The Roundabout (Robo) receptors have been intensively studied for their role in regulating axon guidance in the embryonic nervous system, whereas a role in dendritic guidance has not been explored. In the adult giant fiber system of Drosophila, we have revealed that ectopic Robo expression can regulate the growth and guidance of specific motor neuron dendrites, whereas Robo2 and Robo3 have no effect. We also show that the effect of Robo on dendritic guidance can be suppressed by Commissureless coexpression. Although we confirmed a role for all three Robo receptors in giant fiber axon guidance, the

In the past decade, an array of receptors and ligands have been identified that control growth cone pathfinding and determine axon trajectory (Tessier-Lavigne and Goodman, 1996). The changes in axon trajectory induced by mutations in pathfinding receptors would be expected to have consequences for synaptic connectivity by simply diverting axons toward or away from their targets. However, whether these receptors have guidanceindependent roles in synaptogenesis and the formation of functional synaptic circuitry are not clear. This is attributable in part to technical reasons: it is difficult to record electrophysiologically in the embryo, and it is difficult to separate synaptic effects that are secondary consequences of pathfinding errors from synaptic defects that are attributable to a independent use of receptorligand molecules in synaptogenesis. A role for pathfinding receptors in synaptogenesis may also have been previously overlooked because of the focus on their role in axon guidance.

One of the pathfinding receptor families that has been extensively characterized is known as Roundabout (Robo). In Drosophila, the Robo receptors (Robo, Robo2, and Robo3) and their ligand Slit were first identified and characterized for their role in regulating whether axons cross the midline (Seeger et al., 1993; Kidd et al., 1998a,b, 1999). More recently, the Robos have been

\footnotetext{
Received Sept. 19, 2001; revised Dec. 7, 2001; accepted Jan. 23, 2002.

This work was supported by National Science Foundation Grant IBN9904957 to R.K.M., National Institutes of Health Grant NS18366, and Christopher Reeve Paralysis Foundation Grant GBC1-9801-2 to C.S.G. J.H.S. is a predoctoral fellow and C.S.G. is an investigator with the Howard Hughes Medical Institute. G.J.B. is a recipient of a Burroughs Wellcome Fund career award in the biomedical sciences. We thank Dr. A. Chiba for providing all UAS-comm fly lines, the Spyros ArtavanisTsakonas laboratory for providing the anti-Slit antibody, and the J. Bacon and J. Davies laboratories for the shakB(lethal)-Gal4 line.

Correspondence should be addressed to Dr. Rodney K. Murphey, Department of Biology, Morrill Science Center, University of Massachusetts, Amherst, MA 01003. E-mail: rmurphey@bio.umass.edu.

Copyright (C) 2002 Society for Neuroscience $\quad 0270-6474 / 02 / 223117-13 \$ 15.00 / 0$
}

strong axon guidance alterations caused by overexpression of Robo2 or Robo3 have no effect on synaptic connectivity. In contrast, Robo overexpression in the giant fiber seems to directly interfere with synaptic function. We conclude that axon guidance, dendritic guidance, and synaptogenesis are separable processes and that the different Robo family members affect them distinctly.

Key words: axon; dendrites; guidance; giant fiber; Drosophila; roundabout; robo; slit; synapse; commissureless shown to control the lateral position of axons within the longitudinal tracts (Rajagopalan et al., 2000a,b; Simpson et al., 2000a,b). The robo mutants show ectopic midline crossing, but the position of the longitudinal tracts relative to the midline is undisturbed. The robo2 and robo3 mutants have fewer axons inappropriately crossing the midline, but their longitudinal tracts show braiding and other mispositioning defects along the mediolateral axis. Ectopic expression of Robo in axons that normally project close to the midline has no effect on their lateral positions, but misexpression of Robo 2 or Robo3 in these axons pushes their trajectories away from the midline. Loss-of-function and gain-offunction (GOF) data support the model that a combinatorial code of Robo receptors controls the lateral position of the axons within the longitudinal connectives (Rajagopalan et al., 2000a,b; Simpson et al., 2000a,b).

To assess the role of the Robo receptors in regulating circuit formation in the CNS, we used a system amenable to functional studies. The Drosophila giant fiber (GF) system is responsible for a jump-and-flight response to visual stimuli (Tanouye and Wyman, 1980; Thomas and Wyman, 1984). This system has the advantage that a single pair of descending giant fibers contacts a pair of large target motor neurons [tergotrochanteral motor neuron (TTMn)], and the resulting central synapses can be easily studied anatomically and electrophysiologically in adults. The cell bodies of the GF are located in the brain, and they send dendritic processes into the visual and antennal centers. Each GF extends a single unbranched axon ipsilaterally from the brain to the second thoracic neuromere, where it extends laterally along the dendrites of its target, the TTMn, forming a mixed electrical and chemical synapse (Blagburn et al., 1999). We examined the function of the three Robo receptors by overexpressing each in the GF or its target motor neuron. The results indicate that axon guidance, dendritic guidance, and synaptogenesis are separable pro- 
cesses and that the different Robo family members affect them distinctly.

\section{MATERIALS AND METHODS}

Drosophila stocks. All stocks were grown at $22-25^{\circ} \mathrm{C}$ on standard medium. Two P[GAL]4 lines expressed in the GF system were used. P[GAL4] A307 (Phelan et al., 1996; Allen et al., 1998), hereafter referred to as A307, is a line that shows strong expression in the GF and weak expression in the TTMn and the peripherally synapsing interneuron (PSI). The other line, P[GAL4] c17, hereafter referred to as c17, shows expression in the GF and a subset of sensory neurons (Trimarchi et al., 1999) but in no other identified neurons in the GF system.

The shakB(lethal)-Gal4 line, hereafter referred to as shakB-Gal4, was used to drive expression postsynaptically in the giant fiber. shakB(lethal)Gal4 drives expression in the PSI, the TTMn, and the dorsal longitudinal motor neuron but not in the GF (Jacobs et al., 2000). The following robo upstream-activating sequence (UAS) constructs were used: $U A S$-robo-myc, UAS-robo ${ }^{\triangle C C 2+\triangle C C 3}$-myc, UAS-robo ${ }^{\Delta C C 2}$-myc, UAS-robo ${ }^{\Delta C C 3}$-myc, UASrobo ${ }^{\Delta C}$, UAS-robo (2×), UAS-robo2-myc, UAS-robo2, UAS-robo3, slit ${ }^{2} /$ Cyo, UAS-comm ${ }^{w t}$, and UAS-comm ${ }^{\Delta c}$ (Kidd et al., 1998a,b; Wolf et al., 1998; Bashaw and Goodman, 1999; Bashaw et al., 2000; Simpson et al., 2000a,b).

Immunocytochemistry. CNSs of adults and pupas were dissected in 100 mM phosphate buffer (PB) and immediately fixed in $4 \%$ paraformaldehyde in PB for at least $30 \mathrm{~min}$ at room temperature. Preparations were washed twice in $\mathrm{PB}$, treated with $2 \mathrm{~N} \mathrm{HCl}$ in PBT for 30 min, and further washed four times (10 $\mathrm{min}$ each) to remove the acid. After blocking for $2 \mathrm{hr}$ in PAT (100 mM PB, 1\% bovine serum albumin, and $0.1 \%$ Triton $\mathrm{X}-100)$, the tissue was incubated overnight with a rabbit polyclonal anti $\beta$-galactosidase ( $\beta$-gal) antibody (Cappel, Tunhout, Belgium) at a dilution of 1:6000 in PAT and 3\% normal goat serum. Preparations were then washed at least three times for $1 \mathrm{hr}$ in PAT before incubating with a biotinylated goat anti-rabbit secondary antibody (Vector Laboratories, Burlingame, CA) 1:200 in PAT. Further processing was performed according to $\mathrm{ABC}$ kit instructions (Vector Laboratories). The specimens were dehydrated through a series of ethanol dilutions, cleared using methyl salicylate, and mounted in Canada balsam. For anti-Slit (1:10; Rothberg et al., 1988), anti-Robo (1:50; Kidd et al., 1998a,b), and anti-myc (1:75) staining, a biotinylated horse anti-mouse secondary antibody at a dilution of 1:200 in PAT was used.

Physiology and retrograde staining of the TTMn. Intracellular recordings from muscles were obtained from adult flies in a method similar to that described by Tanouye and Wyman (1980) and Gorczyca and Hall (1984). Flies were anesthetized by cooling down on ice and waxed, ventral side-down, onto a small podium in a Petri dish. The wings were waxed down in an outward position. The GFs were activated extracellularly with brain stimulation by two etched tungsten electrodes, one placed through each eye into the superoesophageal ganglion. A pulse of $\sim 10-20$ $\mathrm{V}$ for $0.03 \mathrm{msec}$ from a Grass S44 stimulator (Grass Instruments, Quincy, MA) was sufficient to give the short latency associated with direct excitation. We therefore routinely gave pulses of $40-60 \mathrm{~V}$ for $0.03 \mathrm{msec}$ to ensure the threshold was always exceeded. For direct extracellular stimulation of the motorneurons, the electrodes were placed into the thoracic ganglion. A tungsten electrode placed in the abdominal cavity served as a ground. Saline-filled glass electrodes pulled to a resistance of 40-60 $\mathrm{M} \Omega$ were driven through the cuticle into the muscle fibers, and intracellular recordings were amplified using a Getting $5 \mathrm{~A}$ amplifier (Getting Instruments, Iowa City, IA).

Each animal was subjected to two standard tests: response latency and following frequency. For latencies, each fly was given 10 single pulses. Measurements were taken from the beginning of the stimulation artifact to the beginning of the EPSP. For following frequency, each animal was given 10 pulses from a Grass S48 stimulator at $100 \mathrm{~Hz}$. The signals were amplified using a Getting 5A microelectrode amplifier and stored on a personal computer with pClamp software and a DMA interface board (Axon Instruments, Foster City, CA). Analysis was performed on a personal computer using pClamp and Excel 97 software (Microsoft, Redmond, WA).

The recording electrode contained $0.5 \%$ neurobiotin, and we injected the TTM muscle iontophoretically at $8-10$ sites for $30 \mathrm{~min}$ in each specimen. Flies were incubated in a moist chamber for $10 \mathrm{~min}$ at room temperature, and the CNS was fixed overnight in $4 \%$ paraformaldehyde in $\mathrm{PB}$ at $4^{\circ} \mathrm{C}$. The motor neuron took up the dye and could be revealed by staining the neurobiotin with the DAB reaction. On many occasions, the TTMn was dye-coupled to the GF axon, and both presynaptic and postsynaptic cells were revealed by staining.

Image capturing and processing. Images in several focal planes were captured from whole-mount CNS preparations using a SPOT digital camera (Diagnostic Instruments Inc., Sterling Heights, MI) and imported into Adobe Photoshop 5.0 software (Adobe Systems Inc., San Jose, CA) on an Apple (Cupertino, CA) Macintosh G3 computer. Montages were then constructed using the "rubber stamp tool" showing axonal projections that cross several planes of focus in the whole-mounted specimen in a single image.

\section{RESULTS}

\section{Wild-type expression of Slit and Robo in pupas and adults}

The GF and the TTMn are thought to be born during the embryonic wave of neurogenesis (Allen et al., 1998, 1999). The GF initiates axonogenesis in the late third instar and has reached the thorax by the beginning of pupation. The GFs make their first contact with the TTMn at $\sim 17 \%$ of pupal development. After reaching the thorax, the GF extends laterally along the TTMn and initiates synaptogenesis during the period from 25 to $50 \%$ of pupal development (Phelan et al., 1996; Allen et al., 1998, 1999). During the remainder of pupal development, the GF continues to grow laterally; the presynaptic and postsynaptic processes grow in diameter as the synapse matures; and gap junctions and chemical synaptic components are put in place.

To determine whether Robo and Slit could be influencing the normal guidance and synaptogenesis of the GF, we used antibodies to determine Slit and Robo expression patterns during various pupal and adult stages during and after GF guidance (Fig. 1). Specific Slit labeling occurs in the midline of the suboesophageal neuromere and in all of the thoracic and abdominal neuromeres presumably on the midline glia (Fig. 1C). Expression of Slit was strongest in early pupas (0-50\% of pupal development) and was not detectable in late pupas (after $75 \%$ ) or in adults. Antibodies to Robo strongly labeled the CNS in a complementary manner; the entire neuropil was labeled with the exception of the midline at all pupal stages (Fig. 1D) and was not detected in adult flies. No specific staining using Robo 2 and Robo3 antibodies could be seen in the CNS in pupae or adults, suggesting that Robo2 and Robo3 are expressed weakly or not at all at these stages (data not shown). However, it should be noted that the antibody to Robo2 is very buffer-sensitive and may not work well in the conditions needed to fix pupal tissues.

\section{Overexpression of Commissureless and a Robo dominant negative reveals an endogenous role for Robos in the GF}

In the embryonic CNS, Commissureless (Comm) functions to downregulate Robo receptors (Tear et al., 1996; Kidd et al., 1998b). Overexpression of Comm mimics the robo loss-offunction phenotype, resulting in ectopic midline crossing. To determine whether Comm expression in the GF would reveal an endogenous role for Robo receptors in GF axon guidance, we expressed $U A S$-comm in the GF using the A307 driver. Expression of Comm alone resulted in a collapse of the GF axons at the midline. The axons often wrap around each other (Fig. 2B, arrow), thereby crossing the midline, whereas the bends of the GF in the target area seem to be normal (Fig. 2B, arrowheads). Consistent with the apparently normal bends, the latency, one measure of synaptic function, was not significantly different from that of control flies (Table 1). Midline crossing of the GF (27\%; $n=22$ ) axons in the target area was also seen when a dominant negative $U A S$-robo ${ }^{\Delta C}$ construct lacking the intracellular domain 
A

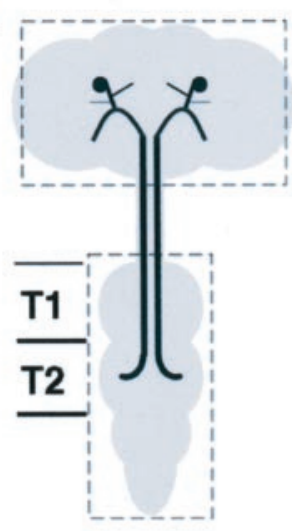

B

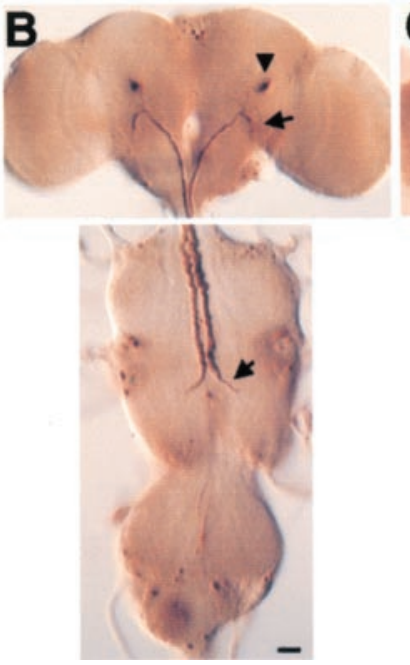

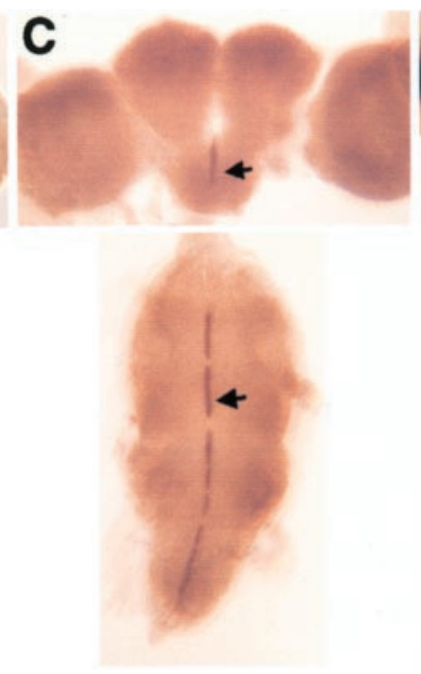

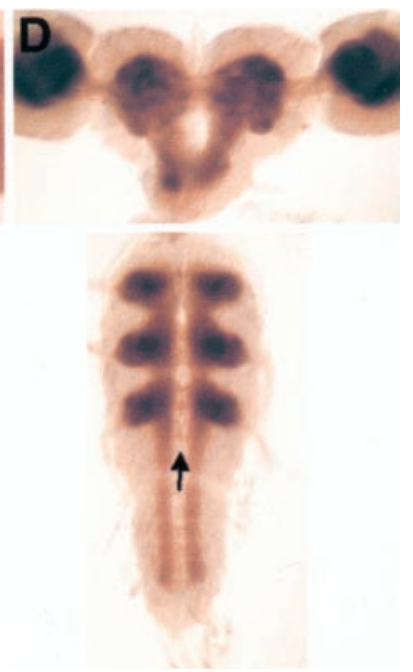

Figure 1. Wild-type expression pattern of Robo and Slit in pupae. $A$, Schematic of the morphology of the GF within the fly CNS. Boxes indicate the regions of the brain and the thoracic ganglion depicted in $B-D$. B. Control adult $(U A S$-lacZ/+;A307/+) CNS whole-mount preparation stained for $\beta$-gal using immunohistochemistry. It displays the wild-type GFs showing their distinct dendritic arbors (top, arrow) and cell bodies (top, arrowhead) in the brain, as well as their axons projecting in T2, where they make a characteristic lateral bend (bottom, arrow). C, CNS whole-mount preparation at $\sim 20 \%$ of pupal development stained for Slit using immunohistochemistry. Note the strong Slit detection in the suboesophageal ganglion and in the thorax localized in the area of the midline glia (arrows). D, CNS whole-mount preparation at $\sim 30 \%$ of pupal development stained for Robo using immunohistochemistry. Note that Robo is detected throughout the neuropil with the exception of weak or no staining at the midline (arrow). Scale bar, $20 \mu \mathrm{m}$.
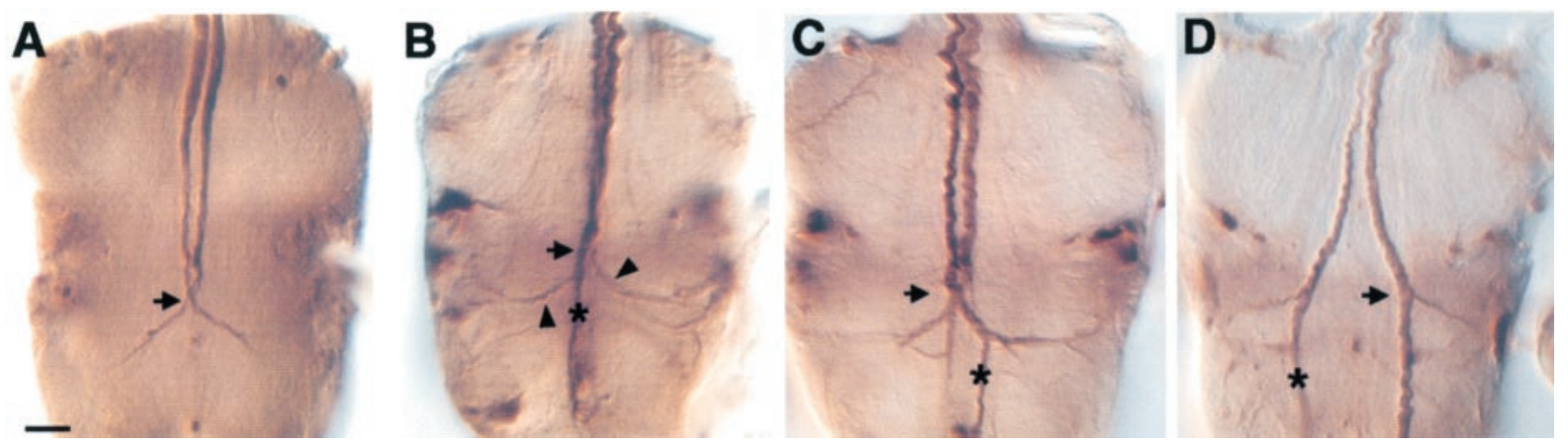

Figure 2. Coexpression of wild-type Comm rescues Robo2-induced lateral displacement. $A$, Expression of dominant negative $U A S$-robo ${ }^{c \Delta}$ using the A307 driver results in a midline crossing of the GF axons in the target area (arrow). B, Expression of UAS-comm ${ }^{w t}$ using the A307 driver results in a collapse of the GF at the midline. The GF axons wrap around each other and cross the midline (arrow). The GF bends appear to be normal (arrowheads). $C$, Coexpression of wild-type UAS-comm and UAS-robo2 using A307 results in GFs with a normal lateral position. In approximately one-third of the specimens, the GF is seen to cross the midline once in the target area, as seen in this specimen (arrow). D, Coexpression of $U A S$-comm ${ }^{c \Delta}$, lacking the intracellular domain, and UAS-robo2 using A307 results in a lateral displacement of the GF, as seen when $U A S$-robo2 alone is expressed in the GF (compare with Fig. 3C). Asterisks indicate that these extensions have been also seen in A307, UAS-lacZ/+ control flies (Allen et al., 1998) and are therefore probably attributable to a nonspecific effect in these recombinant flies. Scale bar, $20 \mu \mathrm{m}$.

(Bashaw and Goodman, 1999) was expressed in the GF by A307 (Fig. 2A). The latency of these specimens was slightly increased in comparison with that of control flies (Table 1). These results are consistent with an endogenous role for Robo in GF axon guidance.

\section{Ectopic expression of Robo, Robo2, and Robo3 in the GF alters axon trajectory}

Overexpression of Robo receptors in the GF reveals that axon trajectory is affected both by the gene being expressed and by the dosage of that particular gene. We used two different p[Gal4] enhancer trap lines, c17 (Trimarchi et al., 1999) and A307 (Phelan et al., 1996; Allen et al., 1998), to target expression of the various robo, robo2, and robo3 UAS constructs to the GF during pupal development and visualized the GF by coexpressing UAS-lacZ and staining for $\beta$-gal. The A307 insertion expresses strongly in the GF and weakly in some of its targets, including the TTMn and the PSI. The c17 insertion drives expression more weakly in the GF but is not expressed postsynaptically in the TTMn motor neuron, allowing determination of phenotypes that are attributable exclusively to presynaptic expression.

When expressed at high levels with the A307 driver, each of the constructs, $U A S$-robo, $U A S$-robo2, and $U A S$-robo3, deflected the GF axon trajectory laterally with $100 \%$ penetrance (Fig. 3, Table 1). Expression of Robo causes the giant axons to deflect mildly in the posterior half of first thoracic neuromere (T1) and in the target area in T2 (Fig. 3B). Robo 2 expression produces an intermediate phenotype. The axons are strongly deflected in the posterior regions of T1 and T2 (Fig. 3C). Finally, Robo3 is able to deflect the GF axons to the extreme lateral edge of the connective in $\sim 15 \%$ of the specimens, and these axons are deflected even 
Table 1. Summary of anatomical and physiological phenotypes induced by Robo, Robo2, and Robo3 expression in the GF

\begin{tabular}{|c|c|c|c|c|c|c|c|c|c|}
\hline \multirow[b]{2}{*}{ Genotype $^{a}$} & \multicolumn{4}{|c|}{ Anatomy } & \multicolumn{5}{|c|}{ Physiology } \\
\hline & $n$ & $\begin{array}{l}\text { Lateral } \\
\text { deflection } \\
\text { (\% GFs) }\end{array}$ & $\begin{array}{l}\text { Lateral } \\
\text { position } \\
(\mu \mathrm{m})\end{array}$ & $\begin{array}{l}\text { ben-like } \\
(\% \text { GFs })\end{array}$ & $n$ & $\begin{array}{l}\text { Latency } \pm \\
\text { SEM (msec) }\end{array}$ & $\begin{array}{l}\text { Normal } \\
\text { synapse } \\
(\% \text { flies })^{b}\end{array}$ & $\begin{array}{l}\text { Weak } \\
\text { synapse } \\
(\% \text { flies })^{c}\end{array}$ & 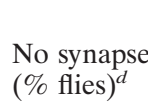 \\
\hline $\mathrm{c} 17 /+$ & 50 & 0 & 1 & 0 & 17 & $0.91 \pm 0.02$ & 100 & 0 & 0 \\
\hline $\mathrm{c} 17 /+; U A S$-robo-myc/+ & 26 & 29 & 1.4 & 14 & 32 & $0.98 \pm 0.11$ & 80 & 22 & 0 \\
\hline $\mathrm{c} 17 /+;(2 \times) U A S$-robo/+ & 50 & 76 & 2.2 & 14 & 53 & $1.23 \pm 0.15$ & 68 & 30 & 2 \\
\hline c17/+;UAS-robo2-myc/+ & 30 & 44 & 1.6 & 0 & 16 & $0.85 \pm 0.03$ & 100 & 0 & 0 \\
\hline $\mathrm{c} 17 /+; U A S-r o b o 3 /+$ & 16 & 57 & 1.9 & 0 & 11 & $0.9 \pm 0.02$ & 100 & 0 & 0 \\
\hline $\mathrm{c} 17 /+; U A S-$ robo $^{\Delta C 2+3} /+$ & 39 & 13 & 1.1 & 0 & 50 & $0.89 \pm 0.06$ & 92 & 8 & 0 \\
\hline $\mathrm{A} 307 /+$ & 50 & 0 & 1 & 0 & 38 & $0.89 \pm 0.02$ & 100 & 0 & 0 \\
\hline $\mathrm{A} 307 /+; U A S$-robo-myc/+ & 33 & 100 & 3.3 & 27 & 22 & $1.81 \pm 0.20$ & 32 & 45 & 23 \\
\hline $\mathrm{A} 307 /+;(2 \times) U A S-r o b o /+$ & 55 & 100 & 3.6 & 26 & 25 & $1.55 \pm 0.26$ & 36 & 36 & 28 \\
\hline $\mathrm{A} 307 /+; U A S-r o b o o^{\Delta C} /+$ & 22 & 0 & 1 & 5 & 13 & $1.02 \pm 0.02$ & 77 & 23 & 0 \\
\hline $\mathrm{A} 307 /+; U A S-r o b o^{\Delta C 2+3} /+$ & 27 & 100 & 3.3 & 4 & 21 & $1.00 \pm 0.10$ & 60 & 31 & 9 \\
\hline $\mathrm{A} 307 /+; U A S-$ robo $2-\mathrm{myc} /+$ & 55 & 100 & 4.7 & 0 & 15 & $0.8 \pm 0.03$ & 100 & 0 & 0 \\
\hline $\begin{array}{l}\text { A307; } U A S \text {-robo2-myc +; } \\
\text { UAS-robo2-myc }\end{array}$ & 20 & 100 & 5.0 & 0 & 13 & $0.92 \pm 0.02$ & 100 & 0 & 0 \\
\hline A307/+;UAS-robo3/+ & 27 & 100 & 5.4 & 0 & 14 & $0.94 \pm 0.08$ & 86 & 14 & 0 \\
\hline $\mathrm{A} 307 /+; U A S-c o m m$ & 20 & 0 & 1 & 0 & 11 & $0.81 \pm 0.03$ & 73 & 27 & 0 \\
\hline
\end{tabular}

${ }^{a}$ For anatomy, the flies also contained a UAS-lacZ either on $\mathrm{X}$ or on the second chromosome.

${ }^{b}$ A normal synapse is defined as response latency $\leq 1 \mathrm{msec}$ and follow stimuli up to $100 \mathrm{~Hz}$.

${ }^{c} \mathrm{~A}$ weak synapse is defined as response latency $>1 \mathrm{msec}$ or do not follow stimuli given at $100 \mathrm{~Hz}$.

${ }^{d}$ These animals showed no response to brain stimulation, but thoracic stimulation of these specimen revealed that the neuromuscular junction response is normal.
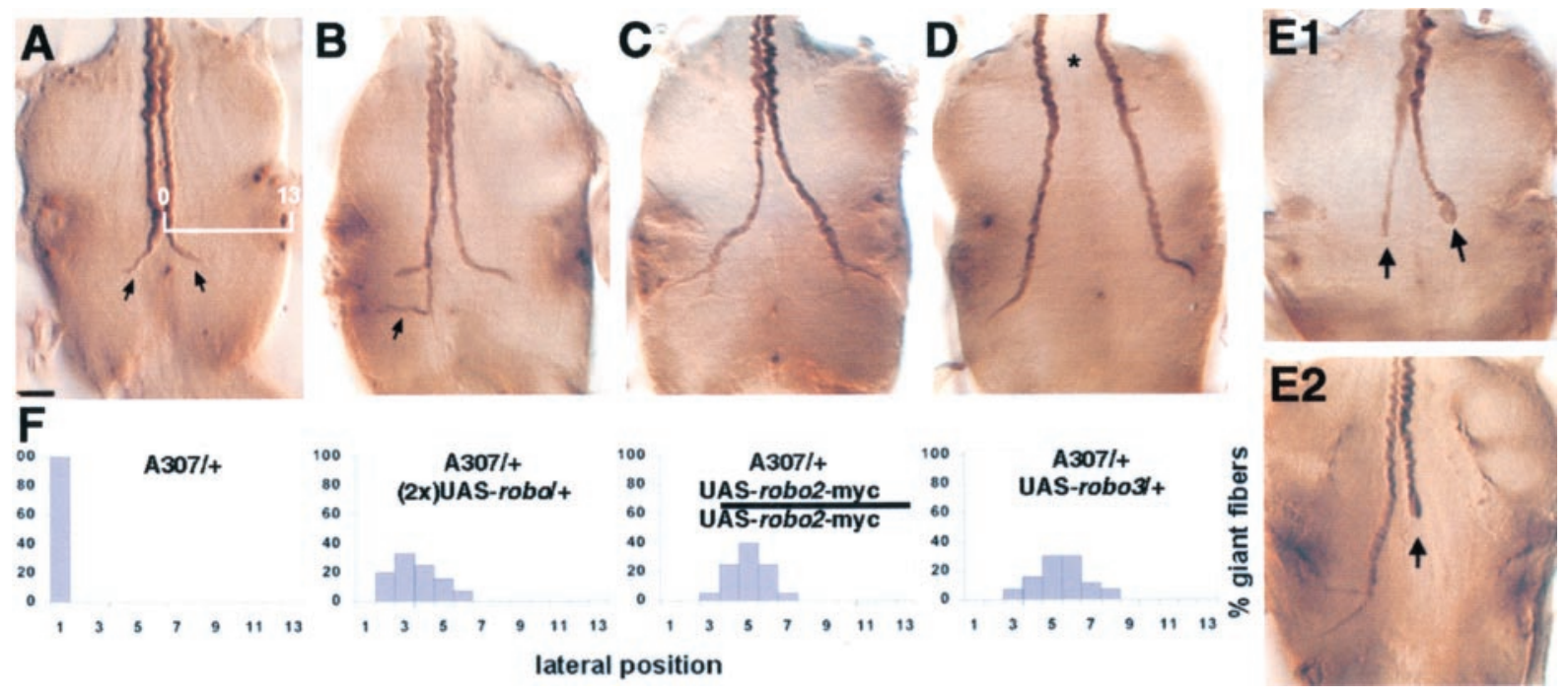

Figure 3. Overexpression of Robo, Robo2, and Robo3 causes lateral displacement of the GF. A, Control adult (UAS-lacZ/+;A307/+) CNS whole-mount preparation stained for $\beta$-gal using immunohistochemistry. This specimen illustrates control GF axons projecting into T2, where they make a characteristic lateral bend (arrows). When $U A S$-robo $(B), U A S$-robo2 $(C)$, and $U A S$-robo3 $(D)$ were expressed in the GF, they revealed a differential strength in their ability to push the GF axons laterally. Note that expression of Robo3 can induce lateral displacement (asterisks) in the connective before reaching the thoracic ganglion. Additional terminal branches of the axon were occasionally observed when $U A S$-robo, UAS-robo2, or UAS-robo3 was ectopically expressed in the giant fiber using either driver $(B$, arrow $)$. E1, ben-like termination in a specimen expressing Robo [A307/+; $(2 \times) U A S$-robo/+] exclusively presynaptically. The ending is swollen or tapered (arrows). E2, ben-like termination in a specimen expressing Robo $[\mathrm{c} 17 /+;(2 \times) U A S-r o b o /+]$. In this case, the ending is swollen (arrows). F, Quantification of the lateral position of the axons. The location of the axon in each specimen was determined relative to the midline and the lateral edge of the ganglion. This distance was divided into 13 intervals, and each GF axon was scored for the relative position of the axon just anterior to the bend (white scale). Black scale bar, $20 \mu \mathrm{m}$.

further laterally as they approach the target area (Fig. 3D). The shift in trajectory to the lateral edge of the connective was never observed with $U A S$-robo or $U A S$-robo2 constructs at any dosage (Fig. 3D, asterisk).

We measured the lateral position of each GF axon with respect to the midline at a single position just anterior to the axon bend.
For this analysis, we divided the CNS into 13 intervals, defining a scale from 0 at the midline to 13 at the lateral edge of the CNS (Fig. $3 A$, white bar). Control axons grew at a position between 0 and 1 just lateral to the midline (Fig. $3 F$ ). To eliminate the possibility that the postsynaptic expression of the A307 driver was affecting the GF trajectory and to clarify the gene dosage effects, 
we expressed the various constructs with the weaker Gal4 driver c17 and quantified the lateral shift of the GF axons. The penetrance with the 17 driver was less complete than with the A307 driver, but the effect of dosage was clarified (Table 1). For example, two copies of $U A S$-robo resulted in a greater lateral displacement than one copy of $U A S$-robo, demonstrating the dosage dependence of the system for Robo.

In contrast, the maximum repulsive output of each receptor is not dependent on dosage. Comparisons between genes confirmed the interactions among dosage, the gene being driven, and the degree of lateralization and eliminated differences in dosage that may exist because of expression levels of each UAS construct. For example, when A307 drives one copy of UAS-robo2, it has a greater repulsive output than two copies of $U A S$-robo, but when the 17 driver was used with the same UAS constructs, the strength of the repulsive output was reversed $(1 \times U A S$-robo 2 in comparison with $2 \times U A S$-robo). Our conclusion, based on the work using both GF enhancers, is that axon trajectory is sensitive to both dosage and the gene being expressed. The various qualitative and quantitative data are consistent in ranking the strength of the repulsive output for axons in the context of the GF: Robo $<$ Robo $2<$ Robo3.

The lateral shifts induced by overexpression of Robo and Robo2 in the GF can be suppressed by co-overexpression of Comm. When UAS-comm was coexpressed with UAS-robo or $U A S$-robo2, the lateral displacement of the GF axons was rescued in $100 \%$ of the cases (Fig. $2 C$; data only shown for Robo2). In some of these preparations, the GF axons cross the midline in the target area, suggesting that Comm expression can completely block the Robo-induced response to Slit (Fig. 2C, arrow). The specificity of this effect was tested by coexpressing a truncated $U A S$-comm ${ }^{\Delta c}$ construct (Wolf et al., 1998), and this had no effect on the Robo- or Robo2-induced deflection (Fig. 2D; example only shown for Robo2).

In contrast to the axonal aberrations induced by expression of additional Robo, Robo2, and Robo3, the dendritic structure of the GF in the brain appeared normal (data not shown). This finding may not be surprising, because the GF dendrites are located in the tritocerebrum, where no slit expression was found (Fig. 1B,C). As will be seen below, this result for the GF is in direct contrast to the results for the motor neuron.

\section{Induction of the bendless-like phenotype}

One of the GF anatomical phenotypes seen with expression of $U A S$-robo but not UAS-robo2 or UAS-robo3 was a "bendless-like" phenotype observed in approximately one-fourth of the GFs using A307 (Fig. 3E1, Table 1) and was only seen in close approximation of the target area but not in the brain or connective. The phenotype varies, because the GF in individual specimens may have a tapered ending similar to the original bendless (ben) mutant phenotype (Muralidhar and Thomas, 1993; Oh et al., 1994), or more often the ending is swollen, as seen when
A

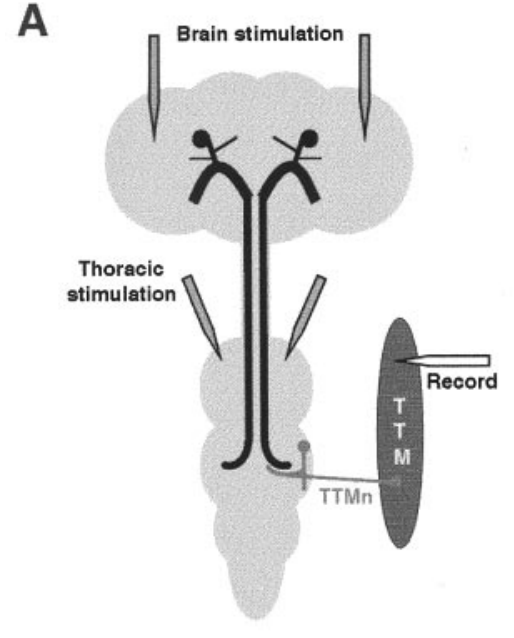

B

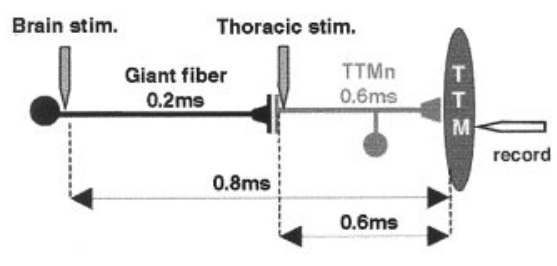

C

C1) Wild type

(Brain)

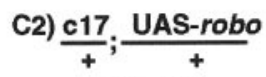

(Brain)

C3)

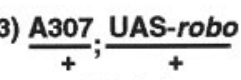

(Brain)
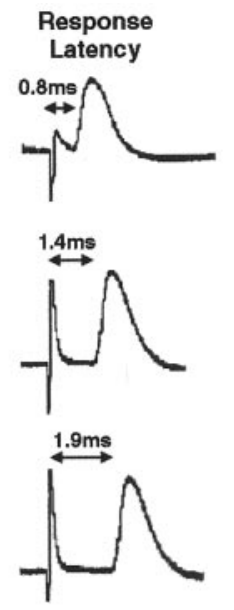

$20 \mathrm{mv}$

$5 \mathrm{~ms}$

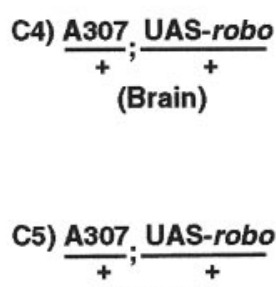

(Thorax)
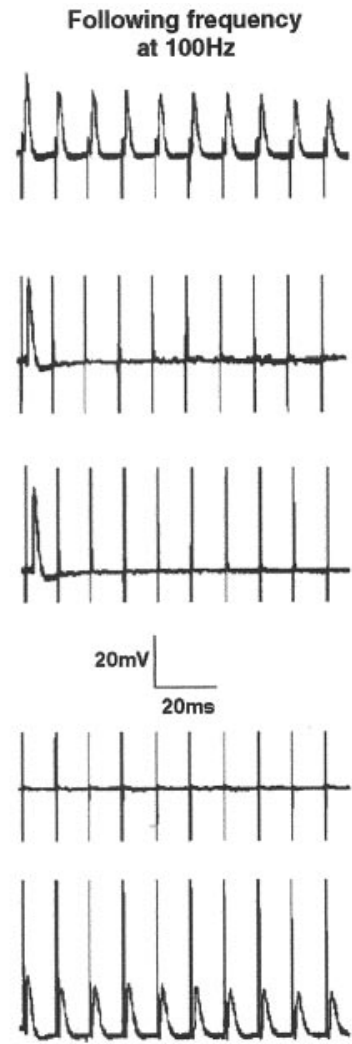

Figure 4. Physiology of the GF circuit. A, Schematic depiction of the two methods of stimulation as well as the method for recording from the TTM muscle. Brain stimulation was used to activate the GF; thoracic stimulation was used to excite the TTMn directly. $B$, Schematic of the GF and the TTMn with approximate conduction times. The estimated response latency is shown for brain $(0.8 \mathrm{msec})$ as well as thoracic stimulation $(0.6 \mathrm{msec}) . C$, Responses of control (C1) and Robo gain-of-function (C2-C5) flies to brain and thoracic stimulation. Note that c17/+;UAS-robo/+ and A307/+;UAS-robo/+ flies have an increased response latency (1.4 and $1.9 \mathrm{msec})$ and are not able to follow repetitive stimulation at $100 \mathrm{~Hz}(C 2$, C3). Some Robo gain-of-function flies $(\mathrm{A} 307 /+; U A S$-robo/+) show no response to brain stimulation $(C 4$, asterisk), but thoracic stimulation reveals that the neuromuscular junction of the TTMn is normal (C5). 
$U A S$-rac or UAS-glued is expressed in the GF (Allen et al., 1999, 2000). Because A307 is expressed both presynaptically and postsynaptically, we thought this ben-like phenotype may be attributable to expression in the motor neurons. However, we also saw the ben-like phenotype when expression was confined to the presynaptic cell (Fig. 3E2, Table 1). Using the weak driver (c17), the probability of the phenotype was lower overall $(14 \%)$, and usually only one of the giant fibers would exhibit the phenotype, whereas the strong driver A307 often affects both GFs, and the overall probability was $27 \%$.

Drosophila Robo has four conserved cytoplasmic motifs that it shares with its homologs in other species (CCO-CC3). The CC2 and $\mathrm{CC} 3$ cytoplasmic motifs that bind to Enabled and Abelson are shared by Robos throughout different species but are not present in Robo2 and Robo3 (Bashaw et al., 2000; Rajagopalan et al., 2000a,b; Simpson et al., 2000a,b). To test the possibility that the $\mathrm{CC} 2$ and $\mathrm{CC} 3$ motifs give Robo the ability to induce the ben-like phenotype, a construct with deleted CC2 and CC3 domains was driven by $\mathrm{A} 307$ or c17. The $U A S$-robo ${ }^{\triangle C C 2 \triangle C C 3}$ construct induced the ben-like phenotype only very rarely, suggesting that these motifs are responsible for this presynaptic phenotype (Table 1). In addition, expression of $U A S$-robo ${ }^{\triangle C C 2}$ and $U A S$ robo ${ }^{\triangle C C 3}$ in the GF using A307 had opposing effects on the penetrance of the ben-like phenotype. Although the lack of the CC2 motif in flies overexpressing mutant Robo resulted in a decrease (from 27 to $8 \% ; n=14$ ), the lack of the CC3 almost doubled the occurrence of the ben-like phenotype in comparison with overexpression of wild-type Robo (from 27 to $44 \%$; $n=16$ ). Interestingly, the lack of the CC2 and the CC3 motifs seemed not to affect the ability of Robo to alter GF axon trajectory (Table 1).

\section{Presynaptic overexpression of Robo affects synaptic function, but Robo2 and Robo3 do not}

We used standard electrophysiological methods (Tanouye and Wyman, 1980) to test the function of the GF synapse in these transgenic specimens. The GF was stimulated in the brain, and recordings were obtained from the TTM muscle (Fig. 4A,B). Two characteristics of the synapse, the latency and the ability of the synapse to follow high-frequency stimulation, were assessed. In wild-type flies, the latency to the brain stimulation is $0.8 \mathrm{msec}$, and the TTMn will follow stimuli at $100 \mathrm{~Hz}$ without failure (Fig. 4C1).

Overexpression of Robo has a large impact on synaptic connectivity. When UAS-robo constructs are driven by A307, approximately one-fourth of the GFs were completely disconnected from the TTMn, and another one-fourth to one-half of the flies show an increased response latency (Fig. 4, Table 1) and are not able to follow stimuli given with a frequency of $100 \mathrm{~Hz}$ (Fig. 4C3, Table 1). The specimens that exhibited no response when the GF was stimulated showed a normal response when the TTMn was stimulated directly, demonstrating that the locus of the defect is the GF $\rightarrow$ TTMn synapse, not the neuromuscular junction (Fig. $4 C 4, C 5)$. In contrast, when Robo 2 or Robo 3 was expressed in the $\mathrm{GF}$, the latencies were normal, and only very subtle defects in following frequency were detected (Table 1). Even with two copies of $U A S$-robo2, we could detect no physiological effect. Apparently the axon trajectory alterations caused by ectopic expression of Robo2 and Robo3 do not affect synaptic connectivity.

There is a strong correlation between the probability of an absent connection and the ben-like anatomy suggesting that these two are related (Table 1). A single copy of $U A S$-robo driven by A307 caused $23 \%$ functional disconnection and $27 \%$ ben-like anatomy. We confirmed this correlation by examining the anat- omy in the same specimens that were disconnected physiologically. In $>75 \%$ of the disconnected specimens, the GF was anatomically ben-like, demonstrating a strong cause and effect between the bendless anatomy and the disconnected physiology. Even the relatively weak expression produced by the c17 enhancer trap line disrupted synaptic transmission and weakened the synapse in approximately one-third of the tested animals. The average response latency was increased, and the synapse was not able to follow stimuli given with a frequency of $100 \mathrm{~Hz}$ (Table 1 , Fig. 4C2). The extreme low probability of a complete disconnection with $\mathrm{c} 17$ can be explained by the low probability of the event and the fact that both GFs are normally connected to both TTMns (Phelan et al., 1996). Therefore, if either GF is intact, the physiological assay does not detect a disconnection.

The response latency of specimens overexpressing $U A S$ robo ${ }^{\triangle C C 2 \triangle C C 3}$ in the GF using A307 was strongly improved, and the penetrance of the disconnection was dramatically reduced in comparison with $U A S$-robo expression (Table 1). However, in $8 \%$ of the investigated specimens, the $\mathrm{GF} \rightarrow \mathrm{TTMn}$ synapse was still defective when $U A S$-robo ${ }^{\triangle C C 2 \triangle C C 3}$ was driven by c17 (Table 1). This suggests that the ben-like phenotype induced by the CC2 and CC3 motifs of the Robo receptor does not account for all weakened synapses seen when full-length robo was expressed in the GF.

We wondered whether the weak synaptic responses seen in specimens expressing Robo or Robo ${ }^{\triangle C C 2 \triangle C C 3}$ (driven by A307 or c17) could be attributed to the GF $\rightarrow$ TTMn connection or were caused by a putative "alternative pathway" (Thomas and Wyman, 1984). We therefore stained the motor neuron of some of these specimens by injecting dye (neurobiotin) at the recording site in the muscle. In control specimens, dye was taken up by the TTMn and transmitted across the gap junction, where it stained the GF, demonstrating the normal dye-coupled contact between the two neurons. We then stained Robo GOF specimens that exhibit a wild-type or a weakened synaptic connection and confirmed that the dye coupling between TTMn and GF was also present in these specimens (Fig. 5B,C). This supports the idea that the weak synapse is in fact a monosynaptic connection and in addition demonstrates that the gap junctions are still present and functional in these defective synapses. The fact that the synapse is weaker than in wild type is presumably caused by the disruption of the impedence matching of the normal electrical synapse. In one case, the GF was not laterally displaced, but a weakened synapse was found, and the GF was still dye-coupled to the TTMn (Fig. $5 B$ ). In a second example (Fig. $5 C$ ), the lateral position of the GF was altered, but the synapse was functionally wild-type, and it was dye-coupled to the TTMn. This result shows that the weakening of the synapse is not correlated with an altered lateral position of the GF axon.

\section{Robo disrupts the dendrites of the TTMn motorneuron, but Robo2 and Robo3 do not}

To investigate the role of the Robo receptors on the postsynaptic neurons, we expressed the various constructs exclusively postsynaptically. We used the shakB-Gal4 line to target expression to the TTMn but not to the GF (Jacobs et al., 2000). In wild-type specimens, the TTMn cell body is located laterally in the second thoracic neuromere and extends two major dendritic branches, one medially and one posteriorly (Fig. 6A). The medial dendrite is the site of the contact with the GF (Fig. $6 A, m d$ ), and the lateral dendrite presumably receives other synaptic inputs from the middle leg (Fig. $6 A, l d$ ). We find $U A S$-lac $Z$ expression by shakB- 

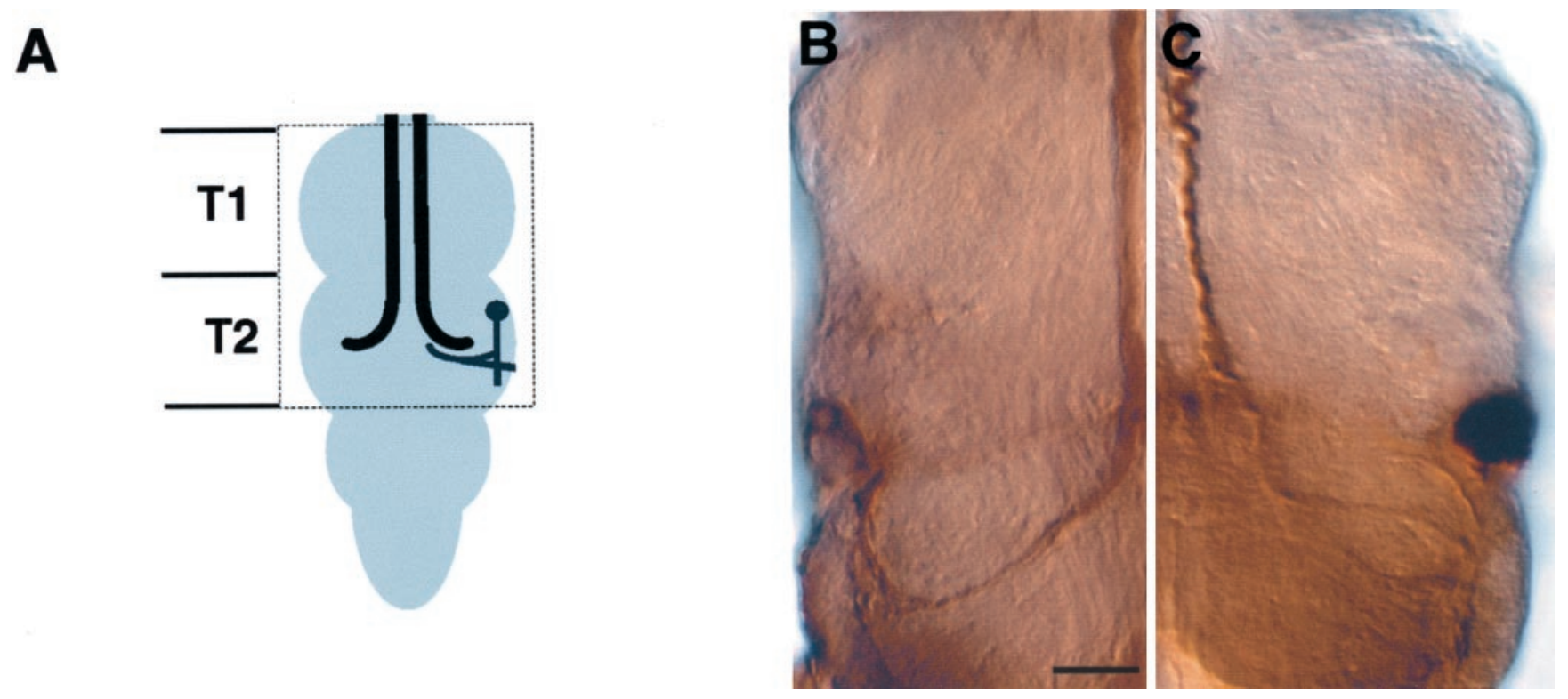

Figure 5. Weak or laterally displaced GF $\rightarrow$ TTMn synapses are dye-coupled. A, Schematic of the morphology of the GF (black) and the TTMn (dark gray) within the thoracic portion of the CNS. The box indicates the regions of the thoracic ganglion depicted in $B, C$. $B$, Retrograde staining of the TTMn in a specimen expressing Robo $[\mathrm{c} 17 /+;(2 \times) U A S$-robo] with a physiologically determined weakened GF $\rightarrow$ TTMn synapse. Note that the TTMn is dye-coupled to the GF. $C$, Specimen expressing Robo [c17/+;(2×)UAS-robo] with a physiologically determined wild-type GF $\rightarrow$ TTMn synapse. Note that the TTMn is dye-coupled to the laterally displaced GF. Scale bar, $20 \mu \mathrm{m}$.

Gal4 to be strongly expressed in the middle stages of pupal development but expressed weakly or not at all in adult flies; therefore, we examined the motor neurons in specimens dissected between 50 and $75 \%$ of pupal development. By this stage, both the GF and the TTMn exhibit their respective bends, are in close apposition, and are in synaptic contact, as indicated by the dye coupling (Phelan et al., 1996; Allen et al., 1998; Jacobs et al., 2000).

Ectopic expression of Robo in the TTMn results in stunted dendrites with a penetrance of $100 \%$ (Table 2, Fig. 6B). The medial dendrites do not reach the midline and appear stalled and distorted 20-30 $\mu \mathrm{m}$ lateral to the midline (Fig. 6B, arrows). The lateral dendrite is often absent or abnormal in these animals as well (Fig. 6B, asterisk). Consistent with the distorted anatomy of the medial dendrite, the physiology of the GF $\rightarrow$ TTMn synapse is weakened but is seldom completely disconnected (Table 2). Typically the latency is increased to $\sim 2 \mathrm{msec}$, and the following frequency is lower in every case. The weak connection suggests that despite the misguidance of the TTMn dendrites, the GF is still able to locate and synapse on its normal target, although the resulting synapse is weaker than usual. In contrast to the results for Robo, the anatomy of TTMn in flies ectopically expressing Robo2 or Robo3 was indistinguishable from that of wild type (Fig. 6C; data only shown for Robo2). Physiologically, expression of UAS-robo2 and UAS-robo3 in the TTM had only subtle effects on the GF $\rightarrow$ TTM connectivity.

Expression of $U A S$-comm or $U A S$-robo ${ }^{\Delta c}$ in the TTMn had no affect on the dendritic projection or the function of the $\mathrm{GF} \rightarrow \mathrm{TTMn}$ synapse (Table 1). This suggests that there is no endogenous role for the Robo receptor in this neuron. However, when we coexpressed Comm and Robo in TTMn, one copy of $U A S$-comm was able to rescue the anatomical and physiological effect of one copy or two copies of UAS-robo, demonstrating the efficiency of the Comm in downregulating the Robo receptor (Table 1). In contrast, coexpression of a truncated $U A S$-comm ${ }^{\Delta c}$ construct (Wolf et al., 1998) had no effect on the ability of Robo to disrupt the dendritic guidance of the TTMn (100\% disrupted dendrites; $n=17$ ). These results suggest that Comm is able to interact and downregulate the Robo receptor not only in axons but also in dendrites.

Because expression of a single copy of UAS-robo in the GF using A307 caused 23\% of the flies to show a disconnection phenotype, and A307 is known to express in the motor neurons, the phenotype in these animals may also be attributable to motor neuron expression (Table 1). However, no disconnection was found when a single copy of $U A S$-robo was expressed strongly and exclusively in the TTMn, suggesting that the weak postsynaptic expression by the A307 driver is not responsible for the disconnection phenotype (Table 2).

\section{The CC2 and CC3 motifs are not responsible for the effect of Robo on dendrites}

To test the possibility that the $\mathrm{CC} 2$ and $\mathrm{CC} 3$ motifs give Robo the ability to affect TTMn dendrite guidance, we expressed UAS $r o b o^{\triangle C C 2 \triangle C C 3}$, in which these two domains are deleted. Despite the domain deletion, expression of UAS-robo ${ }^{\triangle C C 2 \triangle C C 3}{ }_{-m y c}$ resulted in disrupted dendrites with a $100 \%$ penetrance, anatomically and physiologically (Table 2). We also tested constructs that only lack $\mathrm{CC} 2$ or $\mathrm{CC} 3$ as well as a construct that has a Y-F mutation in the CC1 motif (Bashaw et al., 2000), which is shared by all three Robo receptors. The expression of these constructs was not significantly different from that of wild-type UAS-robo in its ability to disrupt the dendritic projection of the lateral TTMn dendrite (data not shown). Therefore, we cannot attribute the difference in dendritic guidance to any of these domains.

\section{Simultaneous presynaptic and postsynaptic overexpression of Robo and Robo2}

To further assess the role of axon pathfinding in the choice of synaptic partners, we examined the synaptic connections made 

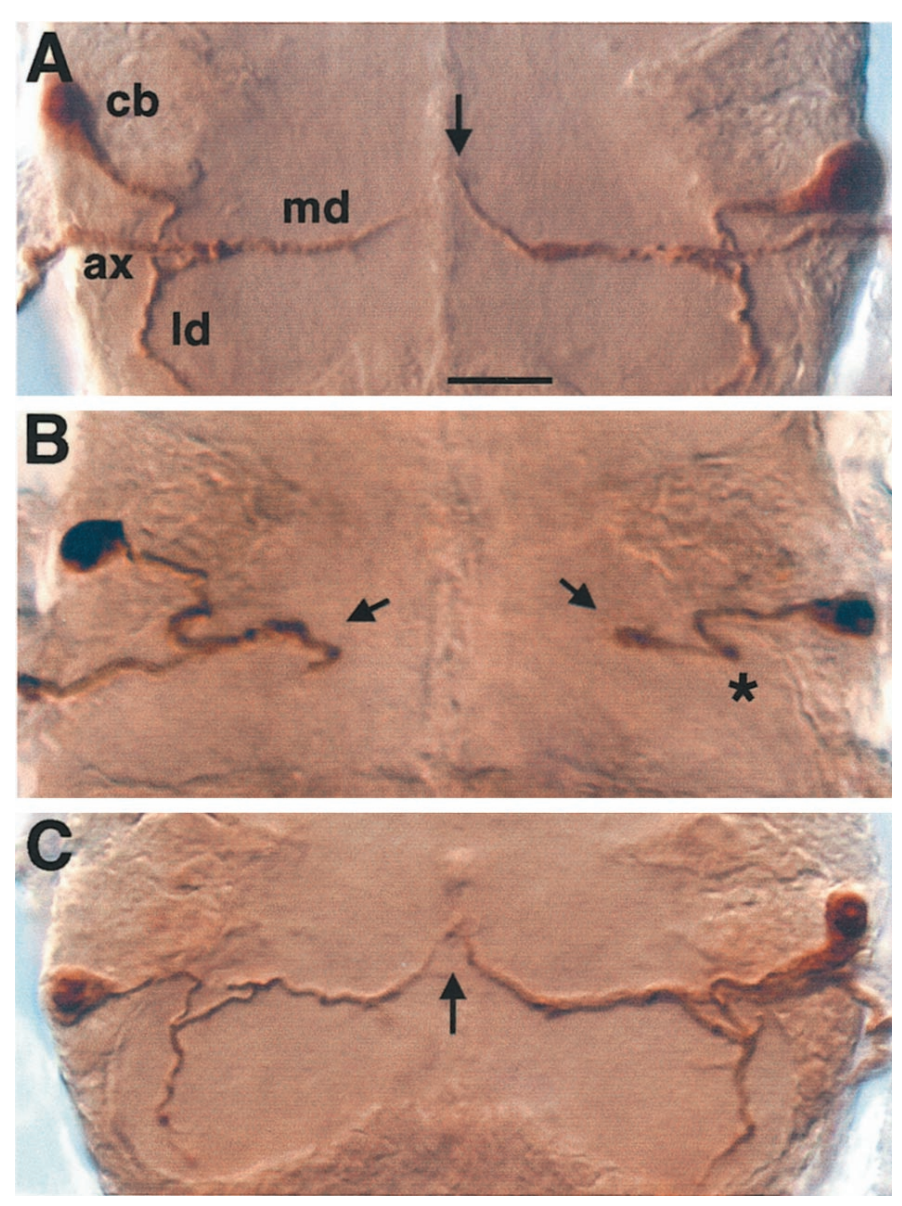

Figure 6. Robo mediates dendritic repulsion of the TTMn, but Robo2 and Robo3 do not. Whole-mount preparations of the thoracic ganglion of late pupas $(50-75 \%)$ were stained for $\beta$-gal using immunohistochemistry. $A$, Example of a control motor neuron (UAS-lacZ/shakB-Gal4). cb, Cell body; $a x$, axon; $l d$, lateral dendrite; $m d$, medial dendrite. $B$, Overexpression of Robo disrupts dendrite formation in the TTMn. The medial dendrite does not reach the midline (arrows), and the lateral dendrite is often missing (asterisk). The genotype is UAS-lacZ/shakB-Gal4;UASrobo-myc/+. $C$, Expression of Robo2 or Robo3 has no effect on the TTMn dendrites (example only shown for UAS-lacZ/shakB-Gal4;UASrobo2-myc/+). As in wild type, the medial TTMn dendrite reaches the midline (arrow). Scale bar, $20 \mu \mathrm{m}$.

when Robo or Robo2 was expressed on both sides of the synapse, in the TTMn and the GF. We combined a GF enhancer (A307 or c17) and the motor neuron enhancer (shakB-Gal4) to visualize the GF and the TTMn simultaneously (examples are shown only for $\mathrm{c} 17 /$ shakB-Gal4). We dissected pupae in the middle of pupal development $(50-75 \%)$ when both drivers are active. Despite the fact that the presynaptic and postsynaptic cells are stained the same color in these experiments, the points of contact are unique sites and are readily identified (Fig. $7 A, B$ ). These unique contact regions are often enlarged (Fig. $7 A$, right $\mathrm{GF}$ ) just as in wild type. In those cases in which we know the contact is completely functional electrophysiologically, such as with robo2 (Table 3, data for A307/shakB-Gal4), these unique contact sites must include the synaptic apparatus. A similar argument applies to the weakened contacts, seen with robo expression; they are unique sites of overlap, although seldom as large as wild type, and functionally they are weaker synapses. If we see no contact, we conclude there is no monosynaptic connection.

An unusually informative specimen is shown in Figure $7 A$. The right side is representative of most specimens, whereas the left side shows one of the numerous variations that occurred at low penetrance. The GF on the right veers laterally as it approaches the target region and contacts the TTMn dendrite in a more lateral position than normal. The region of anatomical contact and overlap between the GF and the TTMn is enlarged, as it would be in wild-type specimens (Fig. $7 A$, arrow on right). The TTMn dendrite can be seen to extend beyond the contact region and reach the midline (Fig. $7 A$, arrowhead on right). A remarkable potential of the GF to compensate for misguidance and eventually to find its target was seen on the left side of the same specimen. The left GF first grew laterally (Fig. $7 A$, white arrows), contacted the TTMn neurite (Fig. $7 A$, white arrowhead), and grew along it to finally contact the medial dendrite and grow along it from lateral to medial (Fig. 7A, left black arrow).

We could detect two competing effects when Robo was overexpressed presynaptically and postsynaptically: an increase in the number of completely wild-type connections and an increase in the number of completely disconnected synapses. When Robo was expressed only in the motor neuron, not a single fly with a wild-type synaptic connection was found, but coexpression both presynaptically and postsynaptically resulted in $22 \%$ wild-type flies (Table 3). This improvement of synaptic connectivity is probably attributable to the lateral deflection of the GF toward the repelled medial TTMn dendrite.

When robo was expressed in the TTMn, we demonstrated that the dendrite never came closer than $20 \mu \mathrm{m}$ from the midline (Fig. $6 B$ ), and yet the GF is usually functionally connected to TTMn, suggesting that the GF must project away from the midline to contact its target. We demonstrated this directly by coexpression of robo both presynaptically and postsynaptically. In one specimen, both GFs extend laterally to reach the displaced dendrite (Fig. $7 B$ ). In another specimen, the right GF extends laterally to contact the dendrite, whereas the left GF terminates near the midline and does not contact the TTMn (Fig. 7C, right arrow). When the GF makes contact in these cases, the contacts are considerably smaller than those of controls, consistent with the weaker physiological connection seen when robo was expressed.

Simultaneous presynaptic and postsynaptic overexpression of Robo also disconnected the GF $\rightarrow$ TTMn synapse more often than expression exclusively presynaptically or exclusively postsynaptically (Table 3). The penetrance of the anatomically ben-like phenotype was increased (42\%) in comparison with expression with the A307 driver (26\%) or the c17 driver alone (14\%). Similarly, the penetrance of the physiological disconnection phenotype was synergistically increased (48\%) compared with that seen when $(2 \times) U A S$-robo was driven only by A307 (27\%) or ShakB-Gal4 (7\%). An example of the ben-like phenotype is seen in Figure $7 C$. The GF on the left shows a swollen ending (black arrow) $\sim 15 \mu \mathrm{m}$ away from the stalled TMMn dendrite (white arrow), whereas the GF on the right contacts TTMn dendrite in an ectopic location.

\section{Distribution of the ectopically expressed Robo-myc and Robo2-myc proteins}

To determine whether the differences observed between the $U A S$ robo-myc and UAS-robo2-myc transgenes were attributable to differential protein distribution, we stained with anti-myc antibody for the ectopically expressed proteins. Three variables influenced the distribution of the ectopic protein: the gene, the neuron examined, and the process (axon or dendrite) examined. The GF axons were stained similarly when the $U A S$-robo-myc and $U A S$-robo2-myc were driven by the A307 line. The axons were uniformly labeled, with a 
Table 2. Summary of anatomical and physiological phenotypes induced by Robo, Robo2, and Robo3 expression in the TTMn

\begin{tabular}{|c|c|c|c|c|c|c|c|}
\hline \multirow[b]{2}{*}{ Genotype $^{a}$} & \multicolumn{2}{|c|}{ Anatomy } & \multicolumn{5}{|c|}{ Physiology } \\
\hline & $n$ & $\begin{array}{l}\text { Disrupted dendrites } \\
\text { (\% TTMs) }\end{array}$ & $n$ & $\begin{array}{l}\text { Latency } \pm \\
\text { SEM (msec) }\end{array}$ & $\begin{array}{l}\text { Normal synapse } \\
(\% \text { flies })^{b}\end{array}$ & $\begin{array}{l}\text { Weak synapse } \\
\text { (\% flies) }^{c}\end{array}$ & $\begin{array}{l}\text { No synapse } \\
\text { (\% flies) })^{d}\end{array}$ \\
\hline ShakB-Gal4/+ & 50 & 0 & 20 & $0.86 \pm 0.02$ & 100 & 0 & 0 \\
\hline ShakB-Gal4/+;UAS-robo-myc/+ & 40 & 100 & 11 & $1.79 \pm 0.16$ & 0 & 100 & 0 \\
\hline ShakB-Gal4/+;(2×)UAS-robo/+ & 21 & 100 & 14 & $2.09 \pm 0.05$ & 0 & 93 & 7 \\
\hline ShakB-Gal4/+;UAS-robo ${ }^{\Delta C 2+3} /+$ & 28 & 100 & 12 & $2.00 \pm 0.21$ & 0 & 83 & 17 \\
\hline ShakB-Gal4/+;UAS-robo2-myc/+ & 20 & 0 & 14 & $0.93 \pm 0.03$ & 100 & 0 & 0 \\
\hline ShakB-Gal4;UAS-robo2-myc & 20 & 0 & 18 & $0.93 \pm 0.02$ & 94 & 6 & 0 \\
\hline \multicolumn{8}{|l|}{ ShakB-Gal4;UAS-robo2-myc } \\
\hline ShakB-Gal4/+;UAS-robo3/+ & 22 & 0 & 14 & $0.91 \pm 0.03$ & 93 & 7 & 0 \\
\hline ShakB-Gal4/+;UAS-robo ${ }^{\Delta C} /+$ & 15 & 0 & 7 & $0.87 \pm 0.01$ & 100 & 0 & 0 \\
\hline ShakB-Gal4/UAS-comm & 20 & 0 & 8 & $0.89 \pm 0.02$ & 100 & 0 & 0 \\
\hline ShakB-Gal4/UAS-comm;UAS-robo/+ & 23 & 0 & ND & ND & ND & ND & ND \\
\hline ShakB-Gal4/UAS-comm; $(2 \times) U A S$-robo/+ & 25 & 0 & 15 & $0.90 \pm 0.02$ & 93 & 7 & 0 \\
\hline
\end{tabular}

ND, Not determined.

${ }^{a}$ For anatomy, the flies also contained a $U A S$-lacZ either on $\mathrm{X}$ or on the second chromosome.

${ }^{b}$ A normal synapse is defined as response latency $\leq 1 \mathrm{msec}$ and follow stimuli up to $100 \mathrm{~Hz}$.

${ }^{c} \mathrm{~A}$ weak synapse is defined as response latency $>1 \mathrm{msec}$ or do not follow stimuli given at $100 \mathrm{~Hz}$.

${ }^{d}$ These animals showed no response to brain stimulation, but thoracic stimulation of these specimen revealed that the neuromuscular junction response is normal.

slight increase of staining intensity near the synaptic terminals (Fig. $8 A 2, B 2$, arrowheads). The GF dendrites were stained weakly (Fig. $8 A 1$, arrow) if at all (Fig. $8 B 1$ ). Increasing the dosage of Robo2myc to match the GF somata staining seen with Robo-myc still failed to stain the GF dendrites (Fig. 8B1), suggesting that the Robo2 receptor is being excluded from the GF dendrites. A307 is an enhancer trap line that drives expression also in some unidentified neurons outside the GF, resulting in a high "background" for the Robo-myc but not for Robo2-myc, and unidentified dendrites are clearly stained in the brain with anti-Robo-myc but not with anti-Robo2-myc (Fig. 8A1,B1).

In the TTMn, the pattern of protein distribution contrasts sharply with the GF, because the dendrites are intensely stained. The overall pattern of protein distribution in the TTMn was similar for Robo-myc and Robo2-myc, but the dendrites consistently stained more intensely than axons or cell bodies (Fig. $8 A 3, A 4, B 3, B 4)$. The level of expression in the GF for Robo-myc staining was similar to that for Robo2-myc (Fig. 8A3,B3). Despite the relatively high levels of Robo2-myc protein in TTMn, the dendrites were not repelled from the midline, whereas less Robo2-myc protein was sufficient to deflect the GF axons from the midline (Fig. 8, compare B2, B4). Because the dendritic guidance effects were so different for the two constructs, we wondered whether this was merely a dosage effect. However, an increase of Robo2-myc dosage did not alter the dendritic structure of the TTMn (Fig. 8B3,B4). It is worth noting that it was difficult to increase the strength of staining of Robo2-myc even with the highest possible dosage. Most of the specimens with multiple copies of the UAS-robo-2-myc transgene (shakB-Gal4/ shak-Gal4;UAS-robo2-myc/+ or shakB-Gal4/+;UAS-robo2-myc/ $U A S$-robo2-myc) show only slight increases in the strength of staining. This suggests that some other variables, such as degradation rates, are controlling protein expression levels, and we have not achieved control of this unknown factor. In summary, these results support the idea that it is not expression dosage that is responsible for the functional difference between Robo and Robo 2 in the regulation of dendritic growth but rather an intrinsic difference between the receptors.

\section{DISCUSSION}

The GF system has allowed us to characterize the differences and similarities between the function of the three Robo receptors in Drosophila and to assess the consequences of their expression on the assembly of an identified synapse. The presynaptic GF axon and the postsynaptic TTMn dendrite respond differently to overexpression of the various Robos. In the GF, as in the embryonic CNS (Rajagopalan et al., 2000a,b; Simpson et al., 2000a,b), all three Robos can shift the axons laterally away from the midline. Misdirecting the GF axons by expression of Robo2 or Robo3 has no effect on synapse function, whereas Robo weakens the $\mathrm{GF} \rightarrow \mathrm{TTMn}$ connection. Our main new finding is that Robo can regulate dendritic growth. In the TTMn, ectopic expression of Robo, but not Robo2 or Robo3, misdirected the dendrites of the motor neuron by deflecting them from the midline, thereby weakening the GF $\rightarrow$ TTMn synapse. The misrouting of the dendrites by Robo can be rescued by Comm coexpression. Overexpression of Robo both presynaptically and postsynaptically highlights the two aspects of Robo function; it restores some synapses to wildtype by compensating for the pathfinding defects but completely disrupts others, suggesting that Robo may also directly interfere with correct synaptogenesis.

\section{Endogenous function of Robo Receptors in the GF}

Considerable evidence suggests that Robo has an endogenous function in guiding the GF axon. First, during pupal development, we could detect the Robo protein throughout the neuropil, and Slit is expressed at the midline. Second, the GF is capable of Slit-mediated repulsion, because overexpression of Robo, Robo2, and Robo3 leads to lateral displacement of the GF. The results are consistent with work in the embryonic CNS in which Robo2 and Robo3 induce a different "final" lateral position than Robo (Rajagopalan et al., 2000a,b; Simpson et al., 2000a,b). Third, the GF grows near the midline but never crosses it. This is similar to the embryonic CNS, in which axons that never cross the midline express Robo receptors on their growth cone and are repelled by Slit expressed by midline glia (Kidd et al., 1998b, 1999). Fourth, ectopic expression of Comm in the GF results in ectopic midline 

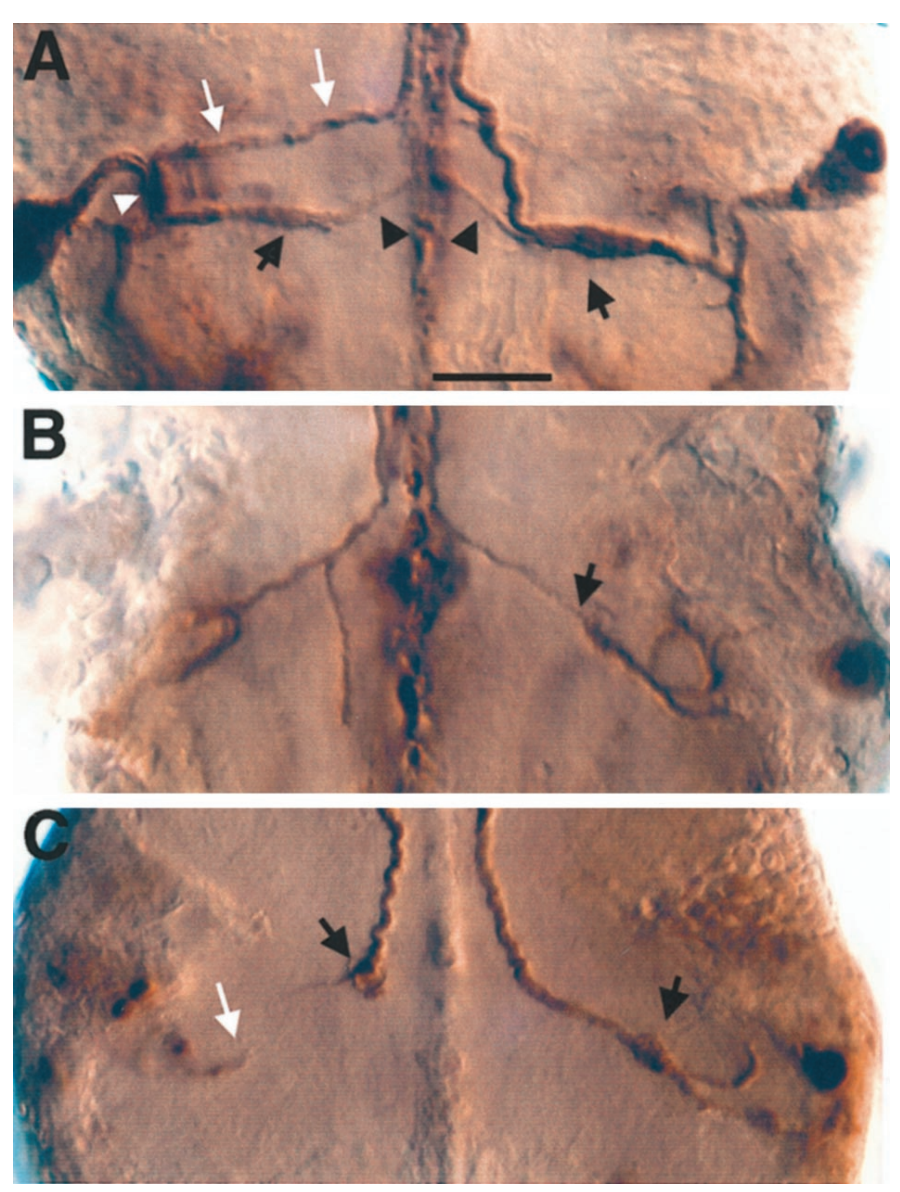

Figure 7. Ectopic GF $\rightarrow$ TTMn synapses in flies expressing Robo or Robo2 presynaptically and postsynaptically. $A$, Simultaneous expression of robo presynaptically and postsynaptically (shakB-Gal4/c17, UAS-lacZ; $U A S$-robo2-myc/+) at $\sim 70 \%$ of pupal development. On the right, the GF contacts the TTMn dendrite more lateral than usual where a thickening of the dendrite and the GF terminal can be seen, presumably representing the ectopic synapse (black arrow). The medial TTMn dendrite extends beyond the contact and reaches the midline (arrowheads). The left GF first grows laterally (white arrows), contacts the TTMn neurite (white arrowhead), and than grows along the medial dendrite from lateral to medial (black arrow). $B, C$, Examples of specimens expressing Robo presynaptically and postsynaptically. Specimens with the genotype shakB-Gal4/ c17,UAS-lacZ; $(2 \times) U A S$-robo dissected at $50-80 \%$ of pupal development are shown. $B$, The GF turns at the midline and grows toward the stunted medial dendrite of the TTMn (black arrow). $C$, In this example, the right GF grows toward and contacts the stalled dendrite $\sim 30 \mu \mathrm{m}$ lateral to the midline (black arrow). The left GF displays a ben-like ending (black arrow) $\sim 10 \mu \mathrm{m}$ from the midline and does not contact the TTMn dendrite (white arrow). Scale bars, $20 \mu \mathrm{m}$.

crossing of the axons, suggesting that Comm may be downregulating endogenous Robo to allow the GF to overcome Slit repulsion. Fifth, overexpression of a dominant-negative Robo in the GF can also result in ectopic midline crossing. All of these results support the idea that one or more of the Robos are required for normal GF guidance by preventing the GF from crossing the midline in the target area.

\section{Role of Robo receptors in dendritic guidance}

A dramatic difference between Robo and Robo2 or Robo3 was revealed when each was expressed in the jump motor neuron (TTMn). Robo had a very powerful effect on the TTMn dendrites, repelling them from the midline, whereas Robo2 and Robo3 had no influence whatsoever on the dendritic projection.
There is complementary evidence from loss-of-function experiments in the embryonic nervous system that Robo has a function in determining the dendritic projection of some motor neurons. In wild-type specimens, the dendrites of the raw prawn 2 (RP2) neuron do not cross the midline, but in the robo loss-of-function mutant, the dendrites do cross the midline (Wolf and Chiba, 2000). The results demonstrate that Robo is involved in the regulation of dendritic projection in this embryonic motor neuron in addition to its well known function in axons. In the case of the adult GF system, we cannot examine loss-of-function mutants easily, because the animals do not survive. We tried to reveal an endogenous role by expressing Comm and Robo ${ }^{\Delta}$, which worked in the axons; however, we could find no evidence for an endogenous role of the Robo receptor in the TTMn. How could these results, suggesting an endogenous role in embryos, and our findings in the adult GF system be integrated? The combined results suggest a model by which neurons could establish their various bilateral and unilateral symmetries. Neurons such as the embryonic RP2 may express Robo to prevent dendrites and axons from approaching or crossing the midline, whereas others may express Robo 2 or Robo3, allowing their dendrites to approach or cross the midline but preventing their axons from approaching or crossing the midline. In this relatively simple manner, the laterality of many neurons in the CNS could be regulated with only a few genes. This would also explain our inability to find an endogenous role for Robo in the TTMn, because Robo in the TTMn would prevent the dendrite from approaching the midline and thereby disrupt connections with the GF.

We considered a number of possible explanations for the functional differences among Robo, Robo2, and Robo3 in dendritic guidance. We were able to show that it is not attributable to differential receptor targeting within the neurons, because no difference in the relative distribution between Robo-myc and Robo2-myc was found. In addition, the functional difference cannot be explained by an obvious difference in their cytoplasmic domains; the CC2 and CC3 motifs are present in Robo but not in Robo2 or Robo3, but their removal in the Robo receptor had no affect on dendritic guidance, suggesting that other motifs in the Robo receptors are responsible for the functional difference. Robo2 and Robo3 may be regulated separately from the regulation of Robo by Comm, and two other comm-like genes have been identified in Drosophila (Rajagopalan et al., 2000a; T. Kidd and C. S. Goodman, unpublished data). If these comm-like genes downregulate Robo2 and Robo3 but not Robo and are endogenously expressed in the TTMn, the difference between the Robo receptors in their ability to affect the TTMn dendritic guidance could easily be explained. This idea that Robo and Robo 2 may be processed differentially is supported by examining the myc-tagged constructs. There seems to be preferential removal of Robo2-myc in the TTMn but not in the GF. When we increased dosage of the gene, the amount of Robo2-myc protein, as indicated by antibody staining of the TTMn axon and dendrites, did not correlate with gene dosage. Additionally, the staining of unidentified neurons outside the giant fiber system is dramatically different for Robomyc and Robo2-myc. Finally, the lack of Robo2-myc staining in GF dendrites suggests that Robo 2 may be degraded or removed preferentially from the surface of dendrites but not axons, whereas Robo is not. In summary, the distinct functions of the Robo receptors may be attributable in part to differential regulation of these proteins at the cell surface.

Although Robo apparently does not function normally in the TTMn, we were able to rescue the Robo-induced misguidance of 
Table 3. Summary of anatomical and physiological phenotypes induced by Robo, Robo2, and Robo3 presynaptic and postsynaptic expression in the GF and the TTMn

\begin{tabular}{|c|c|c|c|c|c|c|c|c|c|}
\hline \multirow[b]{2}{*}{ Genotype $^{a}$} & \multicolumn{2}{|c|}{ Anatomy } & \multicolumn{5}{|c|}{ Physiology } & \multirow[b]{2}{*}{$\begin{array}{l}\text { Weak } \\
\text { synapse } \\
(\% \text { flies })^{c}\end{array}$} & \multirow[b]{2}{*}{$\begin{array}{l}\text { No synapse } \\
\text { (\% flies) }^{d}\end{array}$} \\
\hline & $n$ & $\begin{array}{l}\text { Lateral } \\
\text { deflection } \\
(\% \text { GFs })\end{array}$ & $\begin{array}{l}\text { ben-like } \\
(\% \text { GFs })\end{array}$ & $\begin{array}{l}\text { Disrupted } \\
\text { dendrites } \\
\text { (\% TTMs) }\end{array}$ & $n$ & $\begin{array}{l}\text { Latency } \pm \\
\text { SEM (msec) }\end{array}$ & $\begin{array}{l}\text { Normal } \\
\text { synapse } \\
(\% \text { flies })^{b}\end{array}$ & & \\
\hline $\mathrm{A} 307 /+,(2 \times) U A S-$ robo $/+$ & 55 & 100 & 26 & & 25 & $1.55 \pm 0.26$ & 36 & 36 & 28 \\
\hline ShakB-Gal4/+, $(2 \times) U A S$-robo/+ & 21 & - & - & 100 & 14 & $2.09 \pm 0.05$ & 0 & 93 & 7 \\
\hline ShakB-Gal4/A307, $(2 \times) U A S$-robo/+ & 30 & 100 & 46 & 100 & 23 & $1.64 \pm 0.20$ & 22 & 30 & 48 \\
\hline $\mathrm{A} 307 /+, U A S-r o b o 2-\mathrm{myc} /+$ & 55 & 100 & 0 & & 15 & $0.80 \pm 0.03$ & 100 & 0 & 0 \\
\hline ShakB-Gal4/+, UAS-robo2-myc/+ & 20 & - & - & 0 & 14 & $0.93 \pm 0.03$ & 100 & 0 & 0 \\
\hline ShakB-Gal4/A307, UAS-robo2-myc/+ & 17 & 100 & 0 & 0 & 19 & $0.95 \pm 0.03$ & 100 & 0 & 0 \\
\hline
\end{tabular}

\footnotetext{
${ }^{a}$ For anatomy, the flies also contained a $U A S$-lacZ either on $\mathrm{X}$ or on the second chromosome.

${ }^{b}$ A normal synapse is defined as response latency $\leq 1 \mathrm{msec}$ and follow stimuli up to $100 \mathrm{~Hz}$.

${ }^{c}$ A weak synapse is defined as response latency $>1 \mathrm{msec}$ or do not follow stimuli given at $100 \mathrm{~Hz}$.

${ }^{d}$ These animals showed no response to brain stimulation, but thoracic stimulation of these specimen revealed that the neuromuscular junction response is normal.
}

the TTMn dendrite by Comm coexpression. This demonstrates that the ectopic Robo-Comm machinery can function in dendrites and supports the idea that Robo-Comm interaction may be used to guide dendrites in a manner similar to that seen for axons.

\section{Impact of Robo receptors on synaptic connectivity}

Our results reveal two relatively independent roles for the Robo receptor during synaptogenesis: (1) an indirect regulation of synapse formation by the influence of Robo receptors on anatomical overlap of the axons and dendrites of the two cells; and (2) a direct disruptive effect by weakening the synapse.

There is a powerful effect of the Robos on synaptic connectivity through their regulation of presynaptic and postsynaptic anatomy. When Robo was expressed exclusively postsynaptically, the synapse was weakened in all specimens. This was correlated with the fact that the TTMn dendrites were always pushed laterally, and the GF connections never appeared anatomically normal. However, simultaneous presynaptic and postsynaptic expression could improve the connection so that $22 \%$ of these flies had normal connections. Presumably by pushing the TTMn dendrite and the GF axon laterally, the chances for overlap and strengthening the connection are improved. By regulating the overlap of the axonal and dendritic processes, the Robos control whether the cells are within synaptic grasp of one another, and this provides the outlines of the circuit diagram that will emerge. This may be considered an indirect, although critical, role of the Robo receptors on synaptogenesis.

In addition, Robo appears to have a direct disruptive effect on the $\mathrm{GF} \rightarrow \mathrm{TTMn}$ synapse. A ben-like phenotype was revealed when Robo but not when Robo2 or Robo3 was expressed in the GF. When Robo was expressed in the GF but not in the TTMn, approximately one-third of the specimens exhibited a weakened $\mathrm{GF} \rightarrow \mathrm{TTMn}$ synapse, and half of these were anatomically benlike. However, no ben-like phenotype was found when Robo ${ }^{\Delta C C 2+\Delta C C 3}$ was expressed in the GF, and the synaptic connectivity of the GF $\rightarrow$ TTMn synapse was dramatically improved. Furthermore, we were able to show that in particular the CC2 motif is essential for the induction of the ben-like phenotype. The CC2 and CC3 motifs have been shown to bind to Enable and Abelson, respectively, and to play opposing roles downstream of the Robo receptor (Bashaw et al., 2000). Consistent with these findings, a robo construct lacking the CC3 motif enhanced the occurrence of the ben-like phenotype. The CC2 motif-dependent induction of the ben-like phenotype and the weakening of the $\mathrm{GF} \rightarrow \mathrm{TTMn}$ synapse cannot be simply explained by an altered lateral position of the GF axon because of Robo-induced repulsion from the midline. Robo lacking the $\mathrm{CC} 2$ and $\mathrm{CC} 3$ motifs was still capable of deflecting the GF from the midline. More strikingly, Robo2 and Robo3 are capable of displacing the GF axon even farther from the midline, but the $\mathrm{GF} \rightarrow \mathrm{TTMn}$ synapse in the ectopic location was physiologically completely normal. These results suggest that the presynaptic Robo-induced ben-like phenotype may not be attributable to a pathfinding error but possibly to an interference with target recognition or synaptogenesis. Interestingly, vesl, a member of the vasodilator stimulated phosphoprotein/Ena family in vertebrates, is suggested to play a role in synaptogenesis and synaptic plasticity (Kato et al., 1997). This implies that interfering with endogenous Drosophila Enabled and Abelson signaling by Robo overexpression may have a disruptive effect on synaptogenesis or synapse maturation of the giant fiber.

Simultaneous presynaptic and postsynaptic expression enhanced the penetrance of the ben-like phenotype and the disconnection phenotype, synergistically demonstrating the involvement of the postsynaptic cell in the expression of this phenotype (Table 3). These findings suggest that the presynaptic and postsynaptic partners have found one another, and pathfinding is complete before the emergence of this severe synaptic defect. Furthermore, because simultaneous presynaptic and postsynaptic overexpression is supposed to compensate for the pathfinding errors, because both GF and its TTMn target are shifted laterally, the increase in the number of totally disconnected neurons is likely to be attributable to a synaptic effect rather than the secondary consequence of a guidance defect.

Our interpretation is that the Robo expression on either side of the synapse interferes with synapse formation, but the presence of Robo on both sides synergistically enhances the disruptive effect of Robo on synapse maturation. These results suggest that possibly the Robo receptor needs to be removed from both growth cones and dendrites for synaptogenesis to proceed normally. A similar idea has been proposed by Wolf et al. (1998), who suggested that unknown proteins on the muscle interfere with target recognition or synaptogenesis and need to be removed by Comm. 
A1
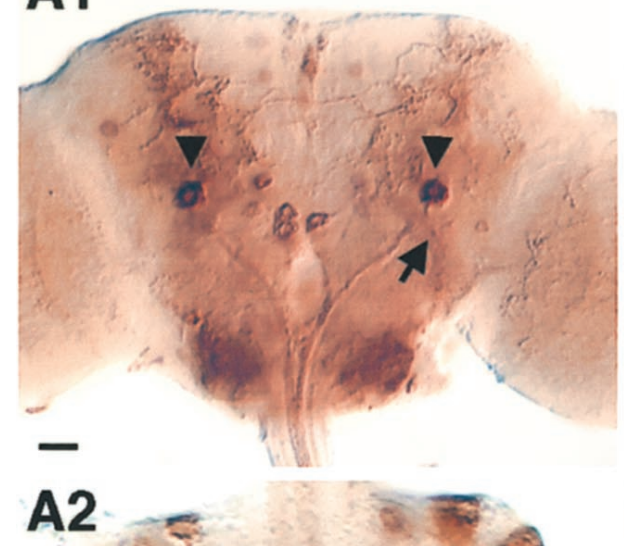

Figure 8. Protein distribution of Robo and Robo2 in the GF and the TTMn. A1, Localization of anti-myc staining when Robo-myc was expressed under the control of A307 (A307/+;UAS-robo-myc/+). Note the staining in the somata (arrowheads) but only very weakly in dendrites (arrow). A2, Robo-myc localization in the axons of the same specimen as in $A 1$. Note the staining in the presynaptic terminal (arrowheads). A3, Robo-myc localization in the motor neurons at $\sim 50 \%$ of pupal development (the genotype is shakB-Gal4/+; $U A S$-robo-myc/+). Note the very dense label in the dendrites (arrows) and the fact that the medial dendrites never reach the midline (asterisk). The axons (arrowhead) and somata are more weakly labeled. A4, Example of Robomyc localization in motor neurons at $\sim 70 \%$ of development (shakB-Gal4/+;UAS-robo-myc/ $+)$. B1, B2, Localization of anti-myc staining when Robo2-myc was expressed by A307 (B1, A307/+;UAS-robo2-myc/+; B2, A307/A307; $U A S$-robo2-myc/+). The somata (B1, arrowheads) and axons are labeled weakly, and the presynaptic terminal is labeled slightly more strongly (B2, arrowheads), but the dendrites could not be detected. Note the increased background and processes of unknown neurons in $A 1, A 2$ in comparison with $B 1, B 2$. B3, Localization of Robo2-myc in the TTMn. Note the strongest labeling in the dendrites (arrows) and the lowest in the axons (arrowhead). Although the label is much weaker, the same differential distribution appears to occur for Robo-myc as for Robo2-myc. The genotype is shakB-Gal4/+;UAS-robo2-myc/+. B4, An increase of Robo2-myc protein in the shakBGal4/shakB-Gal4;UAS-robo2-myc/UAS-robo2myc specimen exhibits the same pattern of expression and, although stronger, still does not deflect the lateral TTMn dendrite from the midline. Scale bars, $20 \mu \mathrm{m}$.
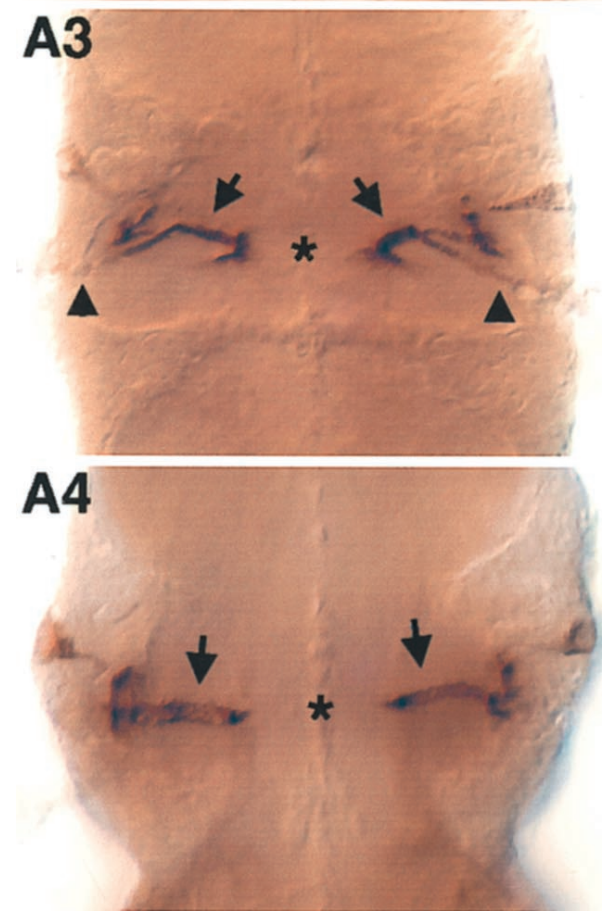
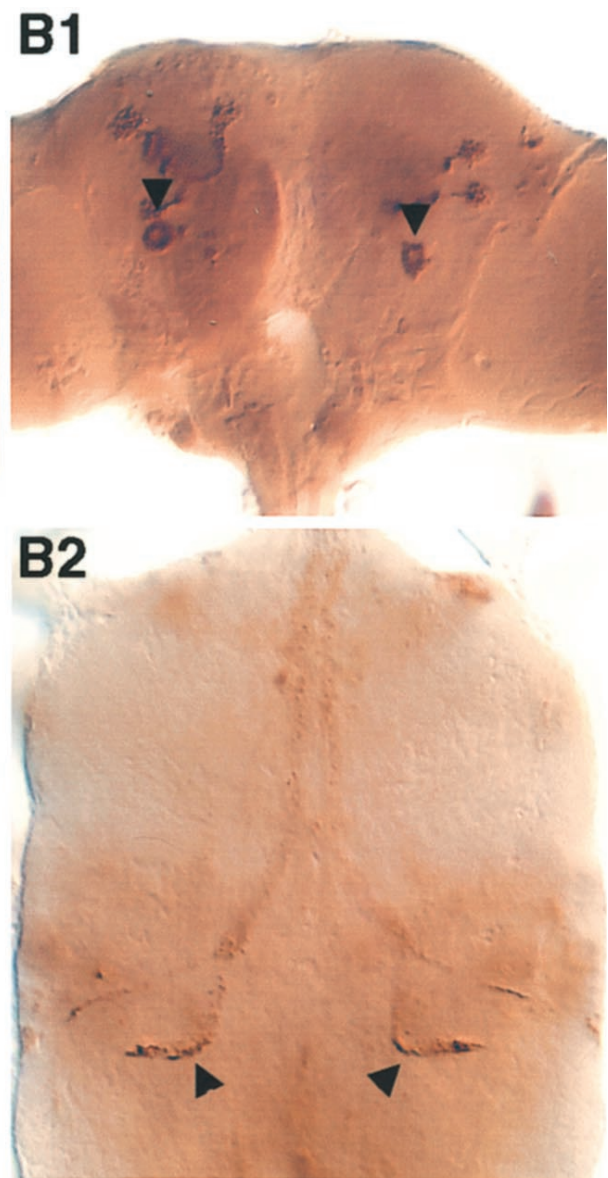
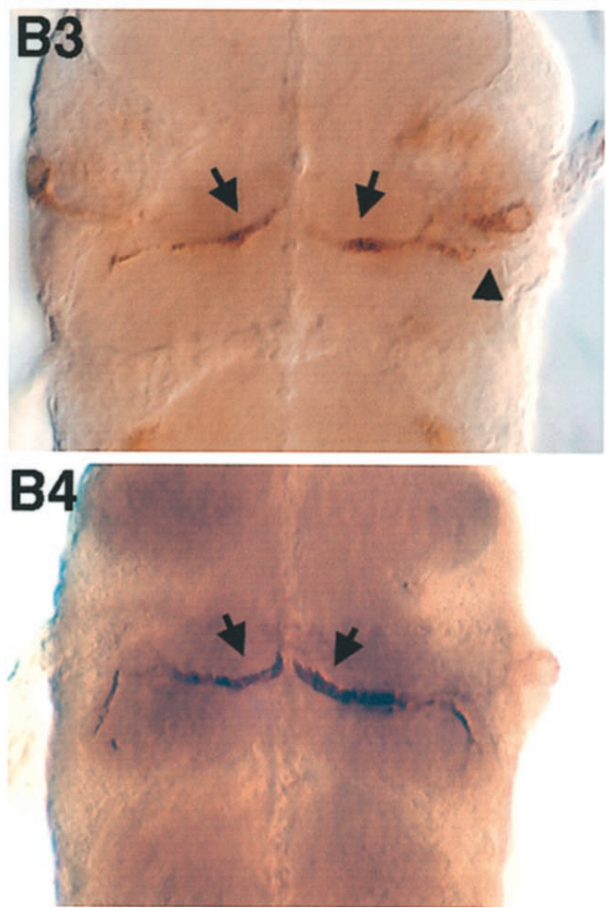

\section{REFERENCES}

Allen MJ, Drummond JA, Moffat KG (1998) Development of the giant fiber neuron of Drosophila melanogaster. J Comp Neurol 397:519-531.

Allen MJ, Shan X, Caruccio P, Froggett SJ, Moffat KG, Murphey RK (1999) Targeted expression of truncated glued disrupts giant fiber synapse formation in Drosophila. J Neurosci 19:9374-9384.

Allen MJ, Shan X, Murphey RK (2000) A role for Drosophila Drac1 in neurite outgrowth and synaptogenesis in the giant fiber system. Mol Cell Neurosci 16:754-765.

Bashaw GJ, Goodman CS (1999) Chimeric axon guidance receptors: the cytoplasmic domains of slit and netrin receptors specify attraction versus repulsion. Cell 97:917-926.

Bashaw GJ, Kidd T, Murray D, Pawson T, Goodman CS (2000) Repul- 
sive axon guidance: Abelson and Enabled play opposing roles downstream of the roundabout receptor. Cell 101:703-715.

Blagburn JM, Alexopoulos H, Davies JA, Bacon JP (1999) Null mutation in shaking-B eliminates electrical, but not chemical, synapses in the Drosophila giant fiber system: a structural study. J Comp Neurol 404:449-458.

Gorczyca M, Hall JC (1984) Identification of a cholinergic synapse in the giant fiber pathway of Drosophila using conditional mutations of acetylcholine synthesis. J Neurogenet 1:289-313.

Jacobs K, Todman MG, Allen MJ, Davies JA, Bacon JP (2000) Synaptogenesis in the giant-fibre system of Drosophila: interaction of the giant fibre and its major motorneuronal target. Development 127:5203-5212.

Kato A, Ozawa F, Saitoh Y, Hirai K, Inokuchi K (1997) vesl, a gene encoding VASP/Ena family related protein, is upregulated during seizure, long-term potentiation and synaptogenesis. FEBS Lett 412:183-189.

Kidd T, Brose K, Mitchell KJ, Fetter RD, Tessier-Lavigne M, Goodman CS, Tear G (1998a) Roundabout controls axon crossing of the CNS midline and defines a novel subfamily of evolutionarily conserved guidance receptors. Cell 92:205-215.

Kidd T, Russell C, Goodman CS, Tear G (1998b) Dosage-sensitive and complementary functions of roundabout and commissureless control axon crossing of the CNS midline. Neuron 20:25-33.

Kidd T, Bland KS, Goodman CS (1999) Slit is the midline repellent for the robo receptor in Drosophila. Cell 96:785-794.

Muralidhar MG, Thomas JB (1993) The Drosophila bendless gene encodes a neural protein related to ubiquitin-conjugating enzymes. Neuron 11:253-266.

Oh CE, McMahon R, Benzer S, Tanouye MA (1994) bendless, a Drosophila gene affecting neuronal connectivity, encodes a ubiquitinconjugating enzyme homolog. J Neurosci 14:3166-3179.

Phelan P, Nakagawa M, Wilkin MB, Moffat KG, O'Kane CJ, Davies JA, Bacon JP (1996) Mutations in shaking-B prevent electrical synapse formation in the Drosophila giant fiber system. $J$ Neurosci 16: 1101-1113.

Rajagopalan S, Nicolas E, Vivancos V, Berger J, Dickson BJ (2000a)
Crossing the midline: roles and regulation of Robo receptors. Neuron 28:767-777.

Rajagopalan S, Vivancos V, Nicolas E, Dickson BJ (2000b) Selecting a longitudinal pathway: Robo receptors specify the lateral position of axons in the Drosophila CNS. Cell 103:1033-1045.

Rothberg JM, Hartley DA, Walther Z, Artavanis-Tsakonas S (1988) slit: an EGF-homologous locus of $D$. melanogaster involved in the development of the embryonic central nervous system. Cell 55:1047-1059.

Seeger M, Tear G, Ferres-Marco D, Goodman CS (1993) Mutations affecting growth cone guidance in Drosophila: genes necessary for guidance toward or away from the midline. Neuron 10:409-426.

Simpson JH, Bland KS, Fetter RD, Goodman CS (2000a) Short-range and long-range guidance by Slit and its Robo receptors: a combinatorial code of Robo receptors controls lateral position. Cell 103:1019-1032.

Simpson JH, Kidd T, Bland KS, Goodman CS (2000b) Short-range and long-range guidance by slit and its Robo receptors. Robo and Robo2 play distinct roles in midline guidance. Neuron 28:753-766.

Tanouye MA, Wyman RJ (1980) Motor outputs of giant nerve fiber in Drosophila. J Neurophysiol 44:405-421.

Tear G, Harris R, Sutaria S, Kilomanski K, Goodman CS, Seeger MA (1996) commissureless controls growth cone guidance across the CNS midline in Drosophila and encodes a novel membrane protein. Neuron 16:501-514.

Tessier-Lavigne M, Goodman CS (1996) The molecular biology of axon guidance. Science 274:1123-1133.

Thomas JB, Wyman RJ (1984) Mutations altering synaptic connectivity between identified neurons in Drosophila. J Neurosci 4:530-538.

Trimarchi JR, Jin P, Murphey RK (1999) Controlling the motor neuron. Int Rev Neurobiol 43:241-264.

Wolf B, Seeger MA, Chiba A (1998) Commissureless endocytosis is correlated with initiation of neuromuscular synaptogenesis. Development 125:3853-3863.

Wolf BD, Chiba A (2000) Axon pathfinding proceeds normally despite disrupted growth cone decisions at CNS midline. Development 127: 2001-2009. 